\title{
СРАВНЕНИЕ АКТИВНО И ПАССИВНО УПРАВЛЯЕМЫХ ИНВЕСТИЦИОННЫХ ФОНДОВ
}

\author{
Володин С.Н. ${ }^{1}$, Кузнеиова М.С. ${ }^{2}$
}

Статья посвящена анализу российского рынка коллективных инвестиций, направленному на определение того, какой тип управления фондом - активный или пассивный является наиболее привлекательным для инвесторов, а именно, приносит большую доходность в долгосрочной перспективе с учетом возможных рисков. Анализ, проведенный за период с 2005 по 2014 г., позволил раскрыть и некоторые иные особенности российского рынка коллективных инвестиций. В результате проведенной в работе оценки динамики доходности фондов были получены выводы об устойчивости результатов управления во времени. Было показано и то, насколько целесообразным является использование инвесторами показателя прошлой доходности фонда для совершения будущих инвестиций. Помимо этого, было изучено, следует ли инвесторам ориентироваться на первую десятку фондов прошлогоднего рэнкинга доходности, либо можно использовать также и фонды, показавшие более низкую доходность.

В целом было установлено, что на российском рынке активным фондам удается лишь незначительно обыграть пассивные с точки зрения доходности в долгосрочной перспективе. Но дополнительный учет рыночного риска, который присущ этим двум типам фондов в разной степени, показал, что пассивно управляемые фонды являются более привлекательными для рядового российского инвестора, особенно если инвестиции в них делаются на несколько лет. В то же время, если инвестор все же решит отдать предпочтение активно управляемым фондам и готов принять на себя дополнительный риск ради получения большей доходности, то ему следует уделить особое внимание диверсификации вложений между различными фондами. Это связано с тем, что устойчивость результатов данного типа фондов во времени невелика, а разброс доходности по ним может достигать существенных величин.

Эти и другие результаты, достигнутые в работе, раскрывают особенности российского рынка коллективных инвестиций и позволят отечественным инвесторам формировать более грамотную политику вложения средств в паевые инвестиционные фонды.

Ключевые слова: инвестиционные стратегии, фонды коллективных инвестиций, доходность вложений, паевые инвестиционные фонды (investment strategies, collective investment funds, return on investment, mutual funds)

JEL: C13 G11

\section{Введение}

Современный фондовый рынок предоставляет инвесторам большой спектр финансовых инструментов и продуктов, позволяющих приумножить собственный капитал. За последние годы большую популярность среди российских инвесторов приобрела такая форма коллективных инвестиций, как паевые инвестиционные фонды (ПИФ). Наиболее высокие темпы роста количества и стоимости чистых активов ПИФ пришлись на период подъема российского рынка до кризиса 2008 г. С каждым годом их количество продолжило увеличиваться и на 2014 г/ составило 1534 фонда, а совокупная стоимость чистых активов возросла до 2124 млрд руб. ${ }^{3}$ Такое развитие рынка паевых фондов обусловлено тем, что они являются одним из наиболее привлекательных, удобных и простых способов размещения капитала для основной массы российских инвесторов. Чтобы использовать данный инструмент, не нужно обладать глубокими финансовыми знаниями. Одновременно с этим стоимость пая относительно невелика, что в свою очередь делает данный вариант инвестирования доступным для широкого круга лиц.

1. Кандидат экономических наук, старший преподаватель Департамента финансов факультета экономических наук НИУ ВШЭ .

2. Проектно-учебная лаборатория анализа финансовых рынков НИУ ВШЭ

3. Обзор ключевых показателей некредитных финансовых организаций по итогам 2014 года / Центральный банк Российской Федерации, май 2015 . URL: http://www.cbr.ru/finmarkets/files/supervision/review_270415.pdf 
По мере роста российского рынка коллективных инвестиций вкладчики все чаще начинают сталкиваться с проблемой выбора оптимального фонда. Анализируя рейтинги ПИФ, можно обратить внимание, что все они отличаются по типу активов: ПИФ акций, облигаций, смешанных инвестиций, индексный и проч. Выбор определенного фонда должен быть во многом обусловлен конкретными целями, которые ставит перед собой инвестор, размещая денежные средства. Большинство пайщиков объединяет основная цель инвестирования - сохранение и преумножение собственных сбережений, но немаловажным фактором при выборе конкретного типа фонда является и отношение вкладчика к риску. Выступая своего рода альтернативой банковским депозитам, вклад в ПИФ позволяет получить доход при относительно небольшой степени риска, по сравнению, например, с вложением средств в акции конкретного эмитента, за счет эффекта диверсификации. Однако риск все же присутствует, поскольку финансовый рынок очень изменчив. Об этом свидетельствует российский индекс волатильности Московской биржи RVI (Relative Vigor Index), максимальное среднемесячное значение которого в 2014 г. составило 47,6\% ${ }^{1}$. Это показывает, что колебание общей доходности многих паевых фондов может быть весьма существенным, и фонд, вложения в который приносят высокий доход сегодня, со временем может оказаться убыточным.

В условиях изменчивости рынка необходимо особенно внимательно подходить к выбору типа фонда, поскольку их инвестиционные стратегии могут сильно различаться. Самое общее деление фондов, между которыми инвестору и предстоит сделать свой выбор, прежде всего - на активно и пассивно управляемые. При активном управлении вкладчик передает право на распоряжение собственными сбережениями конкретным лицам, которые за определенную комиссию совершают операции со средствами фонда, регулярно меняя состав и структуру инвестиционного портфеля с целью получения доходности больше рыночного индекса. Классический пример такого фонда - ПИФ акций. Что касается пассивного управления, то в данном случае состав и структура инвестиционного портфеля соответствуют составу и структуре ценных бумаг, на основе которых рассчитывается один из общепризнанных индексов, поэтому такие фонды получили название индексных [Буренин, 2008]. В отличие от активной стратегии, при пассивной роль управляющих заключается лишь в поддержании данного соответствия.

Выбирая активно управляемый фонд, вкладчик рассчитывает на получение большей доходности, но готов при этом идти и на больший рыночный риск. Более консервативные инвесторы предпочитают индексные фонды, которые, хотя и не позволяют получить доходность, существенно отличающуюся от общерыночной, в то же время не подвержены рискам, связанным с активным управлением.

\section{Актуальность исследования}

Несмотря на то что при активном управлении разработке инвестиционной стратегии уделяется очень серьезное внимание, далеко не всегда это приводит к получению результатов, лучших, чем при пассивном. Западные ученые, анализируя американский финансовый рынок, еще в конце прошлого века пришли к выводу о том, что в среднем активным фондам не удается обыграть пассивные в долгосрочном периоде. Данный вывод был получен, например, в работе М. Грубер [Gruber, 1996]. С 1985 по 1994 г. средний взаимный фонд проигрывал по доходности рыночному индексу на 65 базисных пунктах ежегодно. Похожий вывод был получен и в исследовании Р. Вермерс [Wermers, 2000], где американский рынок взаимных фондов анализируется на протяжении 20-летнего периода (1975-1994 гг.). Было показано, что чистая доходность фондов, в состав которых входили акции, оказалась на $1 \%$ ниже взвешенного по капитализации рыночного индекса CRSP. Общая доходность оказалась выше индекса на 1,3\%, но важно отметить, что при подсчете не учитывались неизбежные для пайщиков операционные расходы и другие транзакционные издержки, которые они несут в случае инвестирования во взаимные фонды такого типа. Подобные тенденции наблюдаются и на современном этапе развития фондового рынка США, о чем говорят регулярно выполняемые исследования

1. URL: http://moex.com/ru/index/RVI 
Standart \& Poor's Indices Versus Active (SPIVA), в которых проводятся сравнения доходности активно управляемых фондов с биржевыми индексами. Согласно данным компании на 31 декабря 2014 г., 86,44\% руководителей фондов большой капитализации не удалось обыграть индекс S\&P. Похожая тенденция наблюдается и на временном горизонте в 5 и 10 лет ${ }^{1}$.

Как показал анализ специализированной литературы, для российского рынка вопрос о том, какие фонды являются более эффективными - активные или пассивные, остается открытым. С одной стороны, это обуславливается относительной молодостью отечественного рынка. С другой - недостатком интереса к данной проблематике в академических кругах. Однако, не имея информации о том, в какой тип фонда более выгодно размещать средства, инвесторам крайне сложно принять грамотное решение. Сегодня на российском рынке коллективных инвестиций наблюдается явный перекос в сторону активно управляемых фондов. За период с 2005 по 2014 г. объем стоимости чистых активов (СЧА) индексных фондов в среднем составлял всего 7,6\% относительно открытых фондов акций. Это говорит о том, что инвесторы рассчитывают на получение большей доходности при выборе активного управления. Но так ли это на самом деле? Получение ответа на этот вопрос и являлось целью данного исследования.

\section{Методология исследования}

Для оценки сравнительной эффективности активно и пассивно управляемых инвестиционных фондов на российском рынке использовались ежегодные значения доходности ПИФов за период с 2005 по 2014 г. Для российского рынка такая длительность периода анализа является максимальной, поскольку, несмотря на то что паевые инвестиционные фонды появились с 1996 г., индексные фонды стали образовываться лишь с января 2003 г. На начало 2005 г. было зарегистрировано семь фондов, из которых работу начали только четыре, что позволило проводить анализ. Для расчетов использовались годовые доходности, а не месячные, что больше соответствует оценке инвестиционных, а не спекулятивных стратегий. В качестве источника информации были использованы рэнкинги доходности ПИФ акций и индексных, предоставляемые порталом investfunds.ru (группа компаний Cbonds).

\section{Вхождение в первую десятку по доходности как критерий выбора фонда инвестором}

Как известно, такая форма коллективного инвестирования, как паевые фонды, ориентирована прежде всего на рядовых, неквалифицированных инвесторов. Как правило, такие инвесторы не обладают глубокими знаниями в сфере финансов и не способны проводить качественный, подробный анализ инвестиционных стратегий, в том числе портфельных. Поэтому основным критерием выбора фонда для них может являться его прошлая доходность. Рэнкинги паевых фондов являются открытой информацией и доступны для основной массы инвесторов, поэтому могут активно использоваться ими.

Конечно, выбирая фонд, рядовой инвестор вовсе необязательно будет ориентироваться только на его прошлую доходность. Выбор может обуславливаться и имиджем управляющей компании, ее известностью, объемом средств в управлении, проводимой рекламной кампанией и прочими факторами. Но все же именно прошлую доходность можно считать основным критерием выбора фонда для потенциального пайщика, так как этот показатель напрямую связан с целью инвестирования - приумножить имеющийся капитал. С другой стороны, с точки зрения анализа того, какие фонды являются наиболее привлекательными для инвесторов, факторы маркетингового и имиджевого характера оценить не представляется возможным ввиду субъективности их восприятия и сложности проведения статистического анализа. Но и говорить, что выбор всецело происходит по одному критерию - доходности, нельзя, так как фактически это означало бы, что инвесторы старались бы разместить свои средства только в те фонды, которые показали наибольшую доходность в прошлом. Поэтому, исходя из того что инвестор при выборе фонда может использовать не только критерий прошлой доходности, но и некоторые другие, можно предположить, что инвесторы чаще всего будут выбирать фонды

1. SPIVA U.S. Scorecard Year-End 2014 / S\&P Dow Jones Indices, 2015. URL: http://www.spindices.com/documents/ spiva/spiva-us-year-end-2014.pdf. 
из первой десятки по доходности, показанной в прошлом.

Для того чтобы понять, является ли нахождение фонда в первой десятке по доходности в прошлом году важным критерием и действительно ли используется в качестве одного из главных для инвесторов при выборе фонда, был проведен анализ динамики средств, привлекаемых в ПИФы. Были рассчитаны средние значения привлеченных средств по активно и пассивно управляемым фондам за период с 2005 по 2014 г. Расчет проводился отдельно для всех фондов по каждому году (n), и для тех фондов, которые вошли в первую десятку рэнкинга доходности в прошлом году (n - 1) (табл. 1). Это позволило оценить, привлекали ли фонды из первой десятки рэнкинга прошлого года средств больше, чем в среднем по всем фондам каждого типа.

Таблица 1

Средний размер привлеченных средств активно и пассивно управляемыми ПИФ за период 2005-2014 гг.

\begin{tabular}{|c|c|c|c|c|c|c|c|c|c|c|c|}
\hline & 2005 & 2006 & 2007 & 2008 & 2009 & 2010 & 2011 & 2012 & 2013 & 2014 & $\begin{array}{c}\text { Сред- } \\
\text { нее }\end{array}$ \\
\hline $\begin{array}{c}\text { 1-я десятка } \\
\text { ПИФ акций, } \\
n-1\end{array}$ & & 949,8 & 265,6 & $-259,9$ & $-113,1$ & 197,8 & -23 & $-12,7$ & 241 & $-487,2$ & 84,3 \\
\hline $\begin{array}{l}\text { Все ПИФ } \\
\text { акций, } n\end{array}$ & 15,2 & 179,9 & $-6,8$ & $-32,6$ & $-30,7$ & $-9,7$ & $-5,5$ & $-54,2$ & $-20,6$ & $-21,5$ & $-0,2$ \\
\hline $\begin{array}{c}\text { 1-я десятка } \\
\text { индексных } \\
\text { ПИФ, } n-1 \\
\end{array}$ & & 277,9 & 11,2 & 18,8 & -12 & 0,3 & -3 & $-22,6$ & $-12,2$ & 13,3 & 30,2 \\
\hline $\begin{array}{c}\text { Все } \\
\text { индексные } \\
\text { ПИФ, } n\end{array}$ & 23,8 & 146,2 & 31,8 & 12,3 & $-18,4$ & $-21,9$ & $-0,3$ & $-36,5$ & $-36,3$ & $-2,5$ & 8,3 \\
\hline
\end{tabular}

Как показывают данные, представленные в таблице, среднее значение привлеченных средств по активно управляемым фондам из первой десятки рэнкинга доходности прошлого года составило 84,3 млн руб., что явно больше аналогичного показателя по всем активно управляемым фондам (-0,2 млн руб.). Та же ситуация наблюдается и для пассивно управляемых фондов: привлечение средств в фонды из первой десятки рэнкинга предыдущего года (30,2 млн руб.) явно больше среднего значения привлеченных средств по всем пассивным фондам (8,3 млн руб.). Такие результаты подтверждают, что инвесторы, размещающие средства в ПИФы, активно используют критерий вхождения фонда в высшие позиции рэнкинга доходности прошлого года, как в случае активно, так и пассивно управляемых фондов.

Проведенные расчеты показали и еще один интересный факт, связанный с поведением инвесторов, разместивших свои средства в активно управляемые фонды из первой десятки прошлогоднего рэнкинга доходности, в ходе кризиса 2008 г. Разразившийся в 2007 г. ипотечный кризис в США и дополнивший его осенью 2008 г. кризис рынка энергоносителей послужили основой для существенного падения цен на отечественном фондовом рынке, в результате чего снижение индекса МІСЕХ (ММВБ) в 2008 г. составило 74\%. На фоне этих событий среднее изъятие средств из фонда для прошлогодней первой десятки активно управляемых составило 259,9 млн руб., в то время как в среднем из активных фондов в данный период изымалось всего 32,6 млн руб. Исходя из этого можно сделать вывод о том, что инвесторы, использующие при выборе фонда критерий доходности предыдущего периода, являются более квалифицированными и лучше реагируют на изменение рыночной ситуации.

Однако это справедливо только для активно управляемых фондов: по пассивно управляемым подобной ситуации не наблюдалось. Это может быть следствием того, что пассивно управляемые фонды относятся к более консервативным инструментам и на них ориентируются пайщики, менее склонные к риску, для которых важно не столько приумножение, сколько сохранение средств. Поэтому они не так гибко реагируют на рыночную ситуацию и более склонны пережидать периоды рыночных падений, ориентируясь на получение положительной доходности в долгосрочном периоде. Как результат - при кризисе 2008 г. масштабного изъятия 
средств из пассивно управляемых фондов не происходило, ни из первой десятки по доходности прошлого года, ни в среднем по фондам такого типа. С другой стороны, некоторые пайщики, изымая средства из более рискованных активно управляемых фондов, могли перекладывать их в течение кризиса в более консервативные, а значит - менее рискованные пассивно управляемые фонды. Поэтому на фоне кризиса для данного типа фондов наблюдался не отток, а даже приток средств.

Важно отметить и тот факт, что инвесторы ориентируются в большей степени именно на первую десятку фондов, показавших максимальную доходность в прошлом периоде. Как показали проведенные расчеты, среднее значение привлеченных средств для фондов из второй десятки по прошлогодней доходности составило 47,3 млн руб., что почти вдвое ниже среднего притока средств в фонды из первой десятки (84,3 млн руб.). Если же исключить влияние рыночного кризиса 2008 г., то на спокойно развивающемся рынке приток средств в фонды из первой десятки по доходности прошлого года составляет уже 127,3 млн руб., еще больше превышая как среднее значение для второй десятки (46,1 млн руб.), так и среднее для активных фондов значение (3,9 млн руб.).

Проведенные расчеты также показали, что по сравнению с активно управляемыми фондами для индексных фондов привлечение средств в первую десятку по доходности предыдущего года ненамного больше среднего значения привлеченных средств по пассивным фондам для текущего года. В данном случае это можно объяснить тем, что индексные фонды занимают меньшую долю российского рынка коллективных инвестиций. Более того, выбирать фонды из первой десятки по доходности имеет смысл именно среди активно управляемых, так как у пассивно управляемых разброс доходности не так велик, и в целом они ближе к доходности рынка. Так что большую роль в случае индексных фондов могут играть иные факторы выбора той или иной управляющей компании.

Итак, можно сделать вывод о том, что инвесторы активно используют доходность предыдущего периода при выборе конкретного фонда для размещения средств. Это выражено для обоих типов рассматриваемых фондов, но более всего проявляется в случае активно управляемых. Поэтому основным объективным критерием выбора фонда для общей массы инвесторов можно считать его расположение на верхних позициях прошлогоднего рэнкинга доходности, а именно в первой десятке. Данный критерий и будет использоваться в работе для сравнения активно и пассивно управляемых фондов.

\section{Сравнение доходности первой десятки активно и пассивно управляемых фондов}

Поскольку инвесторы ориентируются на фонды, показавшие наивысшую доходность в прошлом периоде, направляя в них большее количество средств, возникает вопрос: действительно ли использование данного критерия при выборе фонда является целесообразным и позволяет получать большую доходность от инвестиций? И какой тип фонда является более предпочтительным с точки зрения данного параметра - активно или пассивно управляемый?

Для ответа на этот вопрос сначала попытаемся понять, почему инвесторы склонны выбирать фонды из первой десятки по доходности, показанной в прошлом периоде. Для этого обратимся к графику 1, на котором представлены данные по ежегодной средней доходности для выборок из топ-10 активных и топ-10 пассивных фондов. Можно заметить, что для каждого года фонды, вошедшие в первую десятку, демонстрируют явно большую доходность, чем по всем фондам такого же типа в целом. При этом для активно управляемых фондов эта разница существенно больше, чем для пассивно управляемых: разница в доходности для активно управляемых фондов за период с 2005 по 2014 г. составила 42,7\%, а для пассивно управляемых - всего 3,7\%. Это вполне ожидаемо и объясняется тем, что пассивно управляемые фонды фактически копируют индекс, а значит, их доходность не может сильно отличаться от него. Поскольку у активно управляемых фондов значительно более широкие инвестиционные возможности, то всегда найдутся те из них, которые будут переигрывать динамику общерыночного индекса, которой достаточно точно соответствует доходность индексных фондов.

Ориентируясь на такие высокие показатели доходности первой десятки активно управляемых фондов, рядовой инвестор может предположить, что они являются более доходными и вложение 
средств в них может обеспечить получение большей прибыли в будущем. Поэтому инвесторы так склонны ориентироваться на первую десятку фондов, показавших наибольшую доходность в прошлом, причем именно активно управляемых.

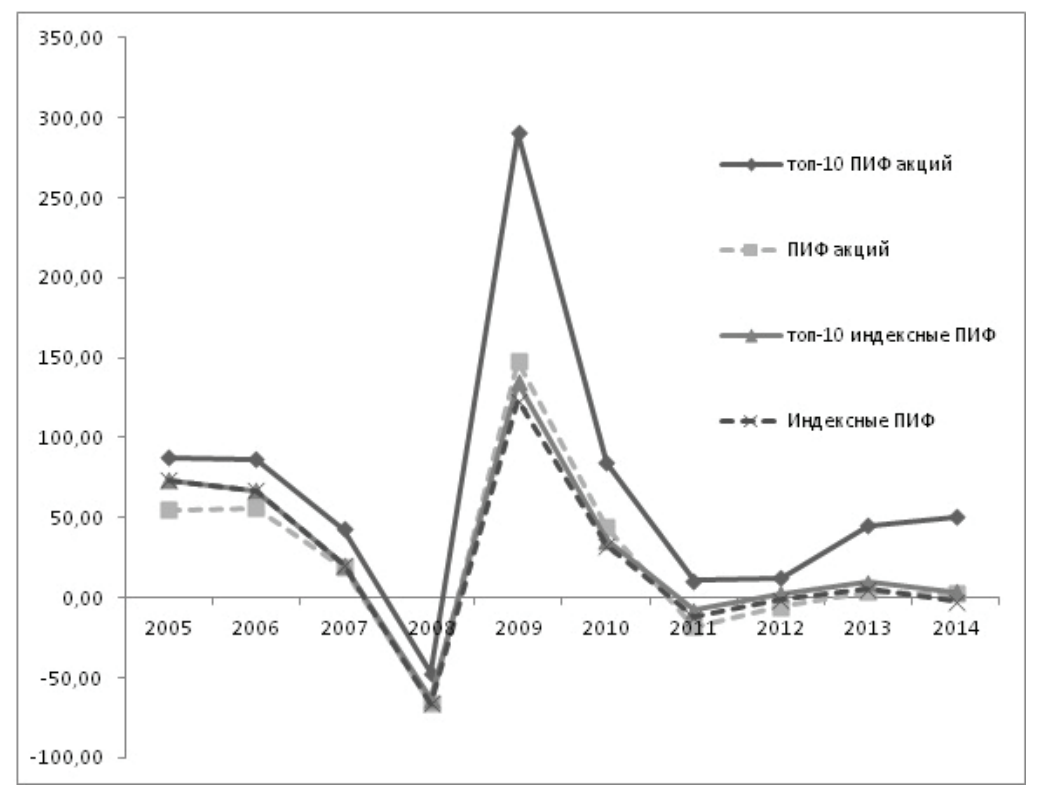

График 1. Средняя доходность топ-10 активных и пассивных фондов, \%

Средняя доходность топ-10 активных и пассивных фондов, \%

\begin{tabular}{|c|c|c|c|c|c|c|c|c|c|c|c|}
\hline & $\mathbf{2 0 0 5}$ & $\mathbf{2 0 0 6}$ & $\mathbf{2 0 0 7}$ & $\mathbf{2 0 0 8}$ & $\mathbf{2 0 0 9}$ & $\mathbf{2 0 1 0}$ & $\mathbf{2 0 1 1}$ & $\mathbf{2 0 1 2}$ & $\mathbf{2 0 1 3}$ & $\mathbf{2 0 1 4}$ & среднее \\
\hline активные & 87,6 & 86,8 & 42,7 & $-47,1$ & 290,5 & 84,4 & 10,8 & 12,4 & 45,2 & 50,8 & 66,4 \\
\hline пассивные & 73,5 & 67,0 & 20,7 & $-63,2$ & 134,6 & 35,9 & $-7,9$ & 2,5 & 9,7 & 3,8 & 27,7 \\
\hline
\end{tabular}

Однако с инвестиционной точки зрения высокие значения доходности фондов из первой десятки вовсе не представляют каких-то гарантий получения такой же высокой доходности в будущем, поскольку нельзя заранее определить, какие именно фонды войдут в десятку лучших по доходности. В связи с этим, более ценной для инвесторов является информация о том, какую доходность способны обеспечить ПИФы, вошедшие в первую десятку текущего рэнкинга доходности, в будущем периоде. Такие данные позволят определить, является ли использование такого критерия, как вхождение фонда в первую десятку по доходности, целесообразным с точки зрения получения большей отдачи от инвестиций в будущем. А сравнение по этому показателю активно и пассивно управляемых фондов покажет, какой тип фонда является более предпочтительным для инвестора с точки зрения достижения большей доходности на вложенный капитал.

Для получения данных выводов, по каждому году из рассматриваемого периода были рассчитаны значения средних доходностей для фондов, вошедших в первую десятку рэнкинга доходности прошлого года. Расчет проводился отдельно для активно и пассивно управляемых фондов (табл. 2). Полученные данные показали, на какой уровень доходности мог бы рассчитывать инвестор, если бы выбирал фонды из десятки лучших по доходности в прошлом году.

Таблица 2

Средняя доходность активно и пассивно управляемых фондов

\begin{tabular}{|c|c|c|c|c|c|c|c|c|c|c|c|}
\hline Год, $\mathrm{n}$ & 2005 & 2006 & 2007 & 2008 & 2009 & 2010 & 2011 & 2012 & 2013 & 2014 & среднее \\
\hline $\begin{array}{c}\mathrm{n}+1, \text { топ-10, } \\
\text { активные }\end{array}$ & & 60,3 & 26,2 & -70 & 94,6 & 73,4 & $-13,4$ & 3 & 22,3 & 0,1 & 21,8 \\
\hline $\begin{array}{c}\mathrm{n}+1, \text { топ- } 10, \\
\text { пассивные }\end{array}$ & & 80 & 19,5 & $-67,2$ & 126,4 & 34 & $-15,3$ & 1,7 & 4,5 & $-4,6$ & 19,9 \\
\hline n, все активные & 54,8 & 56,2 & 18,8 & $-66,3$ & 147,5 & 43,9 & $-18,2$ & $-5,7$ & 3,7 & 2,7 & 20,3 \\
\hline n, все пассивные & 73,5 & 67 & 20,7 & -66 & 123,3 & 32,4 & $-12,3$ & $-1,1$ & 5,2 & $-2,5$ & 18,5 \\
\hline
\end{tabular}




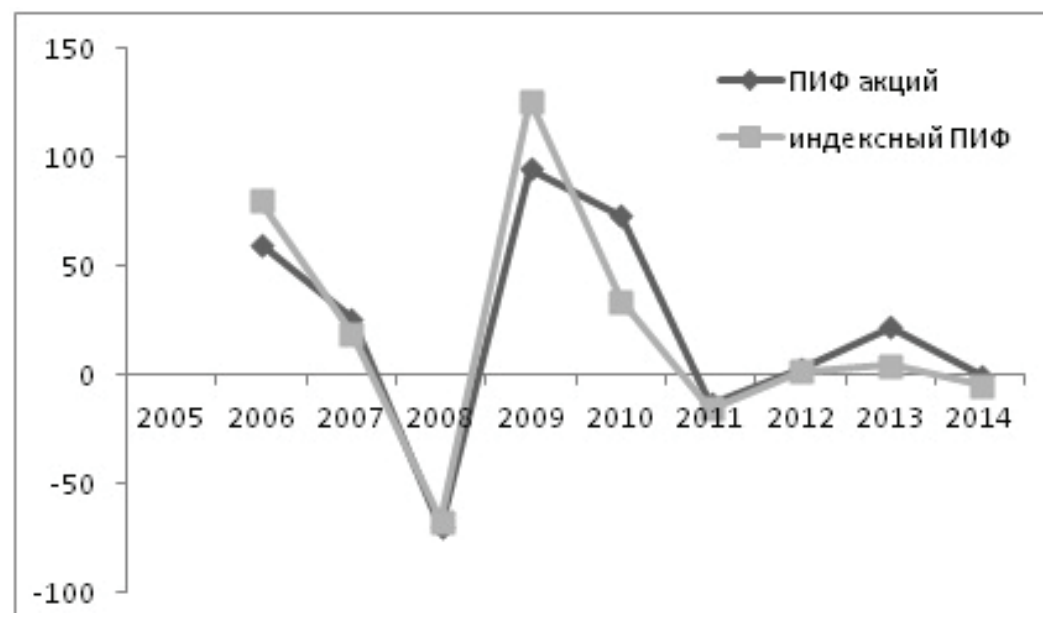

График 2. Средняя доходность первой десятки по доходности активно и пассивно управляемьх фондов прошлого года

Как показывают данные, представленные в таблице 2 и на графике 2, первая десятка активно управляемых фондов по доходности в среднем переигрывает пассивно управляемые на 1,96\%. Поэтому, с одной стороны, активно управляемые фонды способны принести несколько большую доходность по сравнению с пассивно управляемыми. Но с другой стороны, для российского рынка такое превышение доходности нельзя считать значительным. С учетом того что управляющими уделяется существенно большее внимание разработке и реализации инвестиционной стратегии активно управляемых фондов, чем пассивно управляемых, это могло бы способствовать получению большего превышения в доходности.

Проведенные расчеты позволили сделать и другое важное сравнение: позволяет ли прошлогодняя десятка лучших по доходности фондов получать большую доходность относительно всех фондов такого же типа для каждого текущего года. Это дает ответ на вопрос, следует ли инвесторам ориентироваться на прошлогоднюю десятку фондов, занимающих верхние позиции в рэнкинге доходности, при выборе фонда для вложения средств. Как показывает таблица 2, средняя доходность десятки лучших по доходности фондов прошлого года несколько больше, чем для всех фондов в целом, как для активно управляемых (на 1,56\%), так и для фондов с пассивным управлением (на 1,36\%). Поэтому можно сделать вывод, что ориентация на первую десятку фондов и того и другого типа целесообразна и позволяет инвесторам получить несколько большую доходность относительно среднего значения по каждому типу фонда. Однако такое превышение доходности все же нельзя считать большим, оно существенно меньше различия доходности первой десятки рэнкинга и всех фондов каждого типа при сопоставлении по текущему году.

Анализируя доходность инвестиционных фондов, нельзя не затронуть такой показатель, как размер комиссионных издержек, которые несут пайщики. Поскольку анализируемые данные по доходности уже учитывают комиссионные вознаграждения, взимаемые управляющей компанией, депозитарием и другими структурами, входящими в управление фонда, то для проведения более точных сравнений доходности активно и пассивно управляемых фондов необходимо проанализировать только скидки и надбавки, уплачиваемые инвестором при входе и выходе из фонда.

Известно, что при внесении средств в фонд пайщик платит сумму, несколько большую стоимости пая на величину надбавки, а при выводе средств из фонда получает не полную его стоимость, а сниженную на величину скидки. Традиционно считается, что при активном управлении с пайщиков должны взиматься большие размеры комиссий, уплачиваемых при входе и выходе из фонда, ввиду того что оно является более трудоемким процессом, чем пассивное. Однако в российских реалиях существенных различий по данному показателю не наблюдается. Так, например, в 2014 г. для активно управляемых фондов из первой десятки рэнкинга доходности средний размер скидок и надбавок составил соответственно $0,96 \%$ и $0,6 \%$, а для пассивно управляемых - 0,93\% и 0,63\%. Как видно, скидки и надбавки по обоим типам 
фондов являются практически равными, а разница в большей сложности управления активными фондами взимается за счет других видов комиссионных сборов, которые уже учтены в анализируемых показателях доходности. Поэтому полученные в исследовании значения о различии доходности по активно и пассивно управляемым фондам на скидки и надбавки не корректировались.

\section{Правильно ли ориентироваться именно на первую десятку фондов с точки зрения получения большей доходности в будущем?}

Ранее на основе показателя объема привлекаемых средств было показано, что инвесторы ориентируются на первую десятку рэнкинга доходности прошлого года при выборе фонда. Но в ходе исследования было установлено, что и по второй десятке средний размер привлеченных средств был выше, чем в целом по каждому типу фонда. Поэтому интересно оценить, намного ли размер доходности первой десятки фондов превышает доходность второй и третьей десятки. Это позволяет понять, имеет ли смысл инвесторам ориентироваться строго на фонды из первой десятки прошлогоднего рэнкинга доходности, или можно также рассматривать фонды с несколько более низкими позициями.

Поскольку у пассивно управляемых фондов разброс доходности очень незначителен, для ответа на этот вопрос были сделаны расчеты только по фондам с активным управлением. Его результаты представлены в таблице ниже.

Таблица 3

Средняя доходность второй и третьей десятки прошлогоднего рэнкинга активно управляемых фондов

\begin{tabular}{|c|c|c|c|c|c|c|c|c|c|c|}
\hline Год, $\boldsymbol{n}$ & 2006 & 2007 & 2008 & 2009 & 2010 & 2011 & 2012 & 2013 & 2014 & среднее \\
\hline $\begin{array}{c}\text { Вторая } \\
\text { десятка }\end{array}$ & 57,1 & 17,9 & $-68,7$ & 111,5 & 66,4 & $-14,9$ & $-1,8$ & 16,7 & $-2,7$ & 20,2 \\
\hline $\begin{array}{c}\text { Третья } \\
\text { десятка }\end{array}$ & 58,8 & 14,01 & $-64,3$ & 120 & 55,2 & $-27,8$ & $-4,9$ & 14,8 & 5,1 & 19 \\
\hline
\end{tabular}

Как показывают данные из таблицы 3, в среднем для каждого следующего года доходность второй десятки активно управляемых фондов на 1,68\% ниже, чем у первой, а у третьей - уже на 2,86\%. Это говорит о том, что с точки зрения получения большей доходности в следующем периоде первая десятка фондов действительно способна обеспечить лучшие результаты по сравнению со второй и третьей. При этом, если провести сравнение с доходностью всех фондов, то можно увидеть, что в этом случае средняя доходность второй десятки уже является ниже на $0,12 \%$, а третьей - на $1,30 \%$. Это говорит о том, что только первой десятке фондов из прошлогоднего рэнкинга доходности свойственно показывать в следующем периоде результаты лучшие, чем в среднем по всем фондам, поэтому целесообразным можно считать выбор инвесторами фонда только из первой десятки прошлогоднего рэнкинга доходности.

Если оценивать, способна ли вторая или третья десятка прошлогоднего рэнкинга активно управляемых фондов показать лучшие результаты относительно первой десятки пассивно управляемых, то можно отметить, что вторая десятка активно управляемых фондов способна обеспечить лишь крайне незначительное преимущество (в 0,28\%), в то время как доходность третьей десятки будет уже ниже на $0,9 \%$. Поэтому, сравнивая активно и пассивно управляемые фонды, можно говорить о том, что только первая десятка прошлогоднего рэнкинга активно управляемых фондов может обеспечивать некоторое преимущество в доходности.

\section{Диверсификация вложений в активно и пассивно управляемые фонды}

Выше было показано, что первая десятка активно управляемых фондов способна обеспечить большую доходность в будущем периоде, как по сравнению с пассивно управляемыми фондами, так и относительно всех активно управляемых фондов в целом. Но для получения большей доходности инвестор должен поделить свои средства равными долями и распределить 
между всеми фондами, входящими в первую десятку. Однако, как правило, рядовые инвесторы не размещают средства в большом количестве фондов, а склонны выбирать всего один или два фонда. Поэтому важно оценить еще один показатель - насколько устойчивы фонды внутри первой десятки, а именно не является ли разброс их будущей доходности чрезмерно большим. И, выбрав отдельный фонд из первой десятки текущего года, проанализировать, не упадет ли он слишком низко по доходности в следующем году.

Для индексных фондов падение из первой десятки по доходности не столь существенно, как для активных, так как разброс результатов управления по ним не очень велик. Они копируют индекс и не могут сильно отличаться от него по показателям доходности. А вот для фондов с активным управлением падение фондов из первой десятки гораздо более вероятно, поэтому для них был проведен более подробный статистический анализ, показывающий, насколько сильно они склонны перемещаться из верхних позиций рэнкинга.

Результаты анализа представлены в таблице 4. Она показывает, какой процент активно управляемых фондов сохранился в первой десятке по доходности в следующем периоде, сколько переместилось в каждую следующую десятку вплоть до пятой и сколько упало в доходности ниже пятой десятки рэнкинга.

Таблица 4

Изменение ПИФами акций занимаемых позиций в общем рэнкинге (за период с 2006 по 2014 г.), \%

\begin{tabular}{|c|c|c|c|c|c|c|}
\hline Год & $\begin{array}{c}\text { Фонды, } \\
\text { сохранившиеся } \\
\text { в топ-10 }\end{array}$ & $\begin{array}{c}\text { Фонды, } \\
\text { перешедшие } \\
\text { во 2-ой 10 } \\
\text { рейтинга }\end{array}$ & $\begin{array}{c}\text { Фонды, } \\
\text { перешедшие } \\
\text { в 3-ий 10 } \\
\text { рейтинга }\end{array}$ & $\begin{array}{c}\text { Фонды, } \\
\text { перешедшие } \\
\text { в 4-ый 10 } \\
\text { рейтинга }\end{array}$ & $\begin{array}{c}\text { Фонды, } \\
\text { перешедшие } \\
\text { в 5-ый 10 } \\
\text { рейтинга }\end{array}$ & $\begin{array}{c}\text { Фонды, } \\
\text { упвшие ниже } \\
50 \text {-ой позиции }\end{array}$ \\
\hline 2006 & 10 & 20 & 20 & 20 & 30 & 0 \\
\hline 2007 & 30 & 10 & 0 & 40 & 10 & 10 \\
\hline 2008 & 0 & 10 & 0 & 0 & 10 & 80 \\
\hline 2009 & 0 & 0 & 0 & 0 & 10 & 90 \\
\hline 2010 & 60 & 10 & 0 & 10 & 10 & 10 \\
\hline 2011 & 10 & 10 & 10 & 10 & 0 & 60 \\
\hline 2012 & 10 & 30 & 20 & 0 & 10 & 30 \\
\hline 2013 & 30 & 10 & 10 & 20 & 10 & 20 \\
\hline 2014 & 10 & 0 & 0 & 10 & 10 & 70 \\
\hline $\begin{array}{c}\text { Среднее } \\
\text { значение }\end{array}$ & 18 & 11 & 7 & 12 & 11 & 41 \\
\hline
\end{tabular}

Как показывают данные, представленные в таблице, устойчивость активно управляемых фондов в высших позициях рэнкинга доходности невелика. Шансы, что фонд сохранится в первой десятке, достаточно небольшие - всего $18 \%$ фондов сохраняют свое положение на следующий год. Гораздо более вероятно, что фонд переместится из первой десятки на более низкие позиции рэнкинга, вплоть до пятой (41\%), либо с той же вероятностью (41\%) - что займет позиции ниже пятой десятки доходности.

Это хорошо отражает плохую предсказуемость рынков и неустойчивость результатов инвестирования даже у профессиональных управляющих. Причем особенно сильные изменения наблюдаются в периоды сильного падения (2008 г.) и роста (2009 г.) рынка. При больших рыночных флуктуациях управляющим сложнее всего поддерживать высокие результаты, поэтому наблюдаются существенные перестановки фондов в рэнкинге доходности. Так, в 2008 г. 80\% фондов переместились по доходности ниже пятой десятки, а в $2009-90 \%$. Похожая ситуация наблюдалась и при падении рынка в 2011 г., когда $60 \%$ фондов упало ниже пятой десятки рэнкинга.

С другой стороны, как было показано выше, несмотря на существенные перемещения фондов из первой десятки прошлогоднего рэнкинга, в среднем фонды, входившие в нее, все равно способны обеспечить лучшие показатели доходности в будущем периоде, чем фонды, занимавшие более низкие позиции. Поэтому инвесторам имеет смысл ориентироваться на первую 
десятку, но вместе с тем следует больше внимания уделять диверсификации вложений, поскольку каждый отдельный фонд в следующем году может весьма сильно менять позиции внутри рэнкинга доходности и не обеспечить ожидаемую инвестором доходность.

Если же рассмотреть, какая доля пассивно управляемых фондов сохраняется в первой десятке рэнкинга доходности (табл. 5), то можно увидеть, что она значительно выше (57\%), чем у активно управляемых (18\%). Но поскольку разброс доходности у индексных фондов значительно ниже, чем у активно управляемых, данный показатель нельзя отнести к существенным преимуществам пассивно управляемых фондов. Следует отметить и то, что если инвестор выбирает пассивное управление, то для него не существует острой необходимости в диверсификации вложений между разными фондами из первой десятки по доходности, как это имеет место для активно управляемых фондов.

Таблица 5

Доля индексных ПИФ, сохранившихся в первой десятке по доходности

\begin{tabular}{|c|c|c|c|c|c|c|c|c|c|c|}
\hline Год & 2006 & 2007 & 2008 & 2009 & 2010 & 2011 & 2012 & 2013 & 2014 & среднее \\
\hline & $100 \%$ & $100 \%$ & $33 \%$ & $40 \%$ & $60 \%$ & $40 \%$ & $50 \%$ & $50 \%$ & $40 \%$ & $57 \%$ \\
\hline
\end{tabular}

\section{Сравнение активно и пассивно управляемых фондов с точки зрения соотноОшения риска и доходности}

Ранее в ходе анализа активно и пассивно управляемых фондов использовался только показатель доходности, что соответствовало поставленной цели исследования. Но в условиях динамично изменяющегося фондового рынка важную роль играет и учет рисков инвестирования, прежде всего связанных с получением доходности ниже ожидаемого уровня вследствие неблагоприятного изменения цен финансовых активов. Поэтому для того, чтобы более точно оценить, какой именно тип фонда наиболее подходит для инвестирования, были использованы дополнительные показатели, основанные на соотношении риска и доходности.

Известно, что в условиях фондового рынка большей доходности, как правило, соответствует и больший риск. Исходя из этого, при принятии инвестиционных решений необходимо сопоставлять имеющиеся варианты не только с точки зрения доходности на вложенный капитал, но и с учетом рыночного риска неблагоприятного изменения цен. Для проведения подобных сравнений традиционно используются такие показатели, как коэффициент Шарпа и коэффициент вариации. Оба эти коэффициента показывают, какую доходность приносит актив на единицу риска. Чем больше значение коэффициента по рассматриваемому активу, тем больше получит инвестор за принятый на себя риск, а значит - тем более качественным является актив по соотношению риска и доходности. При этом коэффициент Шарпа, в отличие от коэффициента вариации, показывает, покрывается ли более высокий риск актива большей доходностью, за вычетом безрисковой ставки, и не является ли более выгодным вложение средств в безрисковые активы. Значения данных коэффициентов, рассчитанные для каждого текущего года из рассматриваемой выборки по первой десятке прошлогоднего рэнкинга доходности активно и пассивно управляемых фондов, а также стандартное отклонение доходности представлены в таблице ниже.

Таблица 6

Расчет показателей рыночного риска

\begin{tabular}{|l|c|c|c|c|}
\hline & $\begin{array}{c}\text { Коэффициент } \\
\text { Шарпа по первой } \\
\text { десятке, } \mathbf{n + 1}\end{array}$ & $\begin{array}{c}\text { Коэффициент } \\
\text { вариации по } \\
\text { первой десятке, } \mathbf{n + 1}\end{array}$ & $\begin{array}{c}\text { Стандартное } \\
\text { отклонение } \\
\text { доходности по } \\
\text { первой десятке, } \\
\mathbf{n}+\mathbf{1}\end{array}$ & $\begin{array}{c}\text { Стандартное } \\
\text { отклонение } \\
\text { доходности по } \\
\text { всем фондам }\end{array}$ \\
\hline Активно управляемые & $-0,33$ & 0,1 & 18,6 & 20,55 \\
\hline
\end{tabular}


Как показывают полученные данные, несмотря на то что среднегодовая доходность активно управляемых фондов несколько выше пассивно управляемых, первым присуща значительно большая волатильность доходности. Стандартное отклонение доходности активно управляемых фондов в среднем в 2,8 раза выше, чем пассивно управляемых. В результате как с точки зрения коэффициента вариации, так и с точки зрения коэффициента Шарпа более высокий рыночный риск, присущий активно управляемым фондам, не перекрывается большей доходностью по сравнению с пассивно управляемыми. Отрицательная величина коэффициента Шарпа для первой десятки активно управляемых фондов также свидетельствует о том, что больший доход был бы получен при вложении в безрисковые активы, что ставит под сомнение возможность достижения сверхприбыли за счет инвестирования в активные фонды.

С другой стороны, стандартное отклонение по фондам из первой десятки рэнкинга доходности ниже, чем в среднем по фондам каждого типа, как по активно управляемым (на 1,95$)$, так и по индексным (на 4,68). Это характеризует профессионализм управляющих и говорит о том, что выбор фондов из первой десятки прошлогоднего рэнкинга доходности, по сравнению с выбором из всех фондов в целом, имеет смысл и с точки зрения соотношения риска и доходности. Как для активно, так и для пассивно управляемых фондов более высокой доходности в будущем периоде соответствует и более низкий риск.

\section{Основные выводы по работе}

Как показало проведенное исследование, при выборе паевого фонда важным критерием для инвесторов является его присутствие в первой десятке прошлогоднего рэнкинга доходности. То есть инвесторы рассчитывают на повторение успешных результатов управления фондом в будущем.

При этом выбор фондов по наивысшей доходности прошлого года действительно обеспечивает большую доходность в будущем году, по сравнению со всеми фондами такого же типа в целом, к тому же при меньшем риске. Это справедливо как для активно, так и для пассивно управляемых фондов. А значит, квалификация управляющих играет важную роль и придает устойчивость результатам во времени. В случае активно управляемых фондов именно первая десятка рэнкинга приносит большую доходность относительно всех фондов в целом. Что касается второй и третьей десятки, преобладания по доходности относительно всех фондов такого же типа не наблюдалось.

Однако если инвестор предпочитает использовать активно управляемые фонды, следует учесть, что в будущем периоде каждый отдельный фонд из верхушки рейтинга текущего года может весьма сильно упасть вниз, поскольку устойчивость их результатов во времени невелика. В связи с этим инвесторам следует больше внимания уделять диверсификации вложений, выбирая хотя бы несколько фондов из верхушки рейтинга доходности прошлого года.

С точки зрения получения доходности в будущем периоде при вложении средств в первую десятку фондов с наибольшей доходностью прошлого года активно управляемые фонды лишь крайне незначительно обыгрывают пассивные. При этом, если сравнивать с пассивно управляемыми фондами с точки зрения соотношения риска и доходности, более высокий риск активно управляемых фондов в данном случае не перекрывается превышением по доходности. В результате с точки зрения соотношения риска и доходности пассивно управляемые фонды являются более привлекательными для инвесторов. По этому же критерию использование активно управляемых фондов, даже из первой десятки прошлогоднего рэнкинга доходности, нельзя считать целесообразным, так как вложение в них в долгосрочном периоде не позволяет получить доходность выше безрисковой ставки. В результате можно сделать вывод о том, что рядовому инвестору на российском рынке следует ориентироваться на пассивно управляемые фонды, особенно если вложения делаются на долгосрочный период времени. 


\section{Список литературы}

1. Берзон Н.И., Аршавский А.Ю., Буянова Е.А., Красильников А.С. Фондовый рынок. М.: Вита-Пресс, 2009.

2. Берзон Н.И., Володин С.Н. Оценка финансовых активов по критерию «риск-доходность» с учетом длительности инвестирования // Экономический журнал Высшей школы экономики. 2010. № 3, т. 14. С. 311-326.

3. Буренин А.Н. Управление портфелем ценных бумаг. М.: НТО имени академика С.И. Вавилова, 2008.

4. Обзор ключевых показателей некредитных финансовых организаций по итогам 2014 года [Электронный ресурс]: Центральный банк Российской Федерации от 06.05.2015. URL: http:// www.cbr.ru/finmarkets/files/supervision/review_270415.pdf (дата обращения: 22.05.2015).

5. Рейтинги ПИФов по доходности [Электронный ресурс]: данные информационного портала Investfunds группа Cbonds. URL: http://pif.investfunds.ru/funds/rate.phtml (дата обращения: 02.02.2015).

6. Российский индекс волатильности Московской Биржи [Электронный ресурс]: данные Московской Биржи от 01.01.2005. URL: http://moex.com/ru/index/RVI (дата обращения: 10.03.2015).

7. Садкова Ж.А. Паевые инвестиционные фонды как фактор экономического роста // Вестник ДВО РАН. 2006. № 3. С. 29-35.

8. Carhart, M. (2007), “On persistence in mutual fund performance” Journal of Finance, vol. 52, no. 1, pp. 57-82.

9. Davis, J.L. (2001), "Mutual fund performance and manager style", Financial Analysts Journal, vol. 57, no. 1, pp. 19-27.

10. Gruber, M. (1996), “Another puzzle: the growth in actively managed mutual”, Funds. Journal of Finance, vol. 51, no. 3, pp. 783-810.

11. Holmes, M. (2007), "Improved study finds index management usually outperforms active management", Journal of Financial Planning, vol. 20, no. 1, pp. 48-58.

12. Jegadeesh, N., and Titman, S. (1993), "Returns to buying winners and selling losers: Implications for stock market efficiency”, Journal of Finance, vol. 48, no. 1, pp. 65-91.

13. Kremnitzer, K. (2012), "Comparing active and passive fund management in emerging markets". University of California, Berkeley, Economics Department. Senior Honors Thesis.

14. McGuigan, Th.P. (2006), "The difficulty of selecting superior mutual fund performance", Journal of Financial Planning, vol. 19, no. 2, pp. 50-55.

15. Sorensen, E.H., Miller, K.L, and Samak, V. (1998), "Allocating between active and passive management", Financial Analysts Journal, vol. 54, no. 5, pp. 18-31.

16. SPIVA U.S. Scorecard Year-End 2014: S\&P Dow Jones Indices data [Electronic resource]. DOI: http://www.spindices.com/documents/spiva/spiva-us-year-end-2014.pdf.

17. Wermers, R. (2000), "Mutual fund performance: an empirical decomposition into stock-picking talent, style, transactions costs, and expenses", The Journal of Finance, vol. 55, no. 4, pp. 16551695. 


\title{
THE COMPARISON OF ACTIVELY AND PASSIVELY \\ MANAGED INVESTMENT FUNDS
}

\author{
Sergey Volodin, \\ Candidate of Sciences (PhD), Senior Lecturer at the Department of Finance \\ HSE Faculty of Economic Sciences
}

\author{
Maria Kuznetsova, \\ Research \& Training Laboratory of Financial Markets Analysis \\ HSE Faculty of Economic Sciences
}

\begin{abstract}
The article analyzes the Russian market of collective investments aimed at determining what type of fund management - active or passive is the most attractive for investors, specifically, which one brings greater profitability in the long term in view of the possible risks. The analysis carried out for the period from 2005 to 2014 also helped to find out some other features of the Russian market of collective investments. Conclusions about the sustainability of the management over time were obtained as a result of the assessment of the dynamics of the funds' yield. It has been shown how appropriate for investors to use the last rate of return of index of the fund to carry out future investments. In addition, it was investigated whether investors should focus on the top ten ranking last yearss fund returns, or they can also use the funds, which showed a lower rate of return.

In general, it was found that the Russian market actively managed funds only slightly beat passively manages ones in terms of profitability in the long term. However, an additional consideration of market risk showed that passively managed funds are more attractive for the average Russian investor, especially if investments are made over several years. At the same time, if the investor decides to give preference to actively managed funds and is ready to take on additional risk in order to obtain higher yields, he should pay special attention to the diversification of investments between different funds. This is due to the fact that the stability of the results of this type of funds in time is small, and the spread of their yield can reach significant values.
\end{abstract}

These and other achievements in this paper disclose the specifics of the Russian market of collective investments and allow domestic investors to form a competent policy of investing in mutual funds.

Keywords: investment strategies, collective investment funds, return on investment, mutual funds

JEL: C13 G11

\section{References}

1. Berzon N.I., Arshavskij A.Ju., Bujanova E.A., Krasil'nikov A.S.; Fondovyj rynok [Stock market]; M.:Vita-Press, 2009.

2. Berzon N.I., Volodin S.N.; Ocenka finansovyh aktivov po kriteriju «risk-dohodnost'»s uchetom dlitel'nosti investirovanija [Valuation of financial assets on the criterion of 'riskreturn' considering the duration of the investment]; Jekonomicheskij zhurnal Vysshej shkoly jekonomiki. 2010. № 3, t. 14. S. 311-326.

3. Burenin A.N.; Upravlenie portfelem cennyh bumag [Portfolio management.]; M.: NTO imeni akademika S.I. Vavilova [NTO Academician S.I. Vavilov]; 2008.

4. Obzor kljuchevyh pokazatelej nekreditnyh finansovyh organizacij po itogam 2014 goda [Jelektronnyj resurs]: Central'nyj bank Rossijskoj Federacii ot 06.05.2015. [Review key indicators of non-credit financial institutions at the end of 2014 [electronic resource]: The Central Bank of the Russian Federation of 05.06.2015]; URL: http:/www.cbr.ru/finmarkets/ files/supervision/review_270415.pdf (data obrashhenija: 22.05.2015).

5. Rejtingi PIFov po dohodnosti [Jelektronnyj resurs]: dannye informacionnogo portala Investfunds gruppa Cbonds [Ratings of mutual funds according to their yield [electronic resource]: Data Portal Investfunds group Cbonds]; URL: http://pif.investfunds.ru/funds/rate. 
phtml (data obrashhenija: 02.02.2015).

6. Rossijskij indeks volatil'nosti Moskovskoj Birzhi [Jelektronnyj resurs]: dannye Moskovskoj Birzhi ot 01.01.2005 [Russian Volatility Index of the Moscow Stock Exchange [electronic resource]: the data of the Moscow Stock Exchange on 01.01.2005]; URL: http://moex.com/ ru/index/RVI (data obrashhenija: 10.03.2015).

7. Sadkova Zh.A.; Paevye investicionnye fondy kak faktor jekonomicheskogo rosta [Mutual funds as a factor of economic growth]; Vestnik DVO RAN. 2006. № 3. C. 29-35.

8. Carhart, M. (2007), “On persistence in mutual fund performance” Journal of Finance, vol. 52 , no. 1 , pp. 57-82.

9. Davis, J.L. (2001), "Mutual fund performance and manager style", Financial Analysts Journal, vol. 57, no. 1, pp. 19-27.

10. Gruber, M. (1996), “Another puzzle: the growth in actively managed mutual”, Funds. Journal of Finance, vol. 51, no. 3, pp. 783-810.

11. Holmes, M. (2007), "Improved study finds index management usually outperforms active management", Journal of Financial Planning, vol. 20, no. 1, pp. 48-58.

12. Jegadeesh, N., and Titman, S. (1993), "Returns to buying winners and selling losers: Implications for stock market efficiency", Journal of Finance, vol. 48, no. 1, pp. 65-91.

13. Kremnitzer, K. (2012), "Comparing active and passive fund management in emerging markets". University of California, Berkeley, Economics Department. Senior Honors Thesis.

14. McGuigan, Th.P. (2006), "The difficulty of selecting superior mutual fund performance", Journal of Financial Planning, vol. 19, no. 2, pp. 50-55.

15. Sorensen, E.H., Miller, K.L, and Samak, V. (1998), "Allocating between active and passive management”, Financial Analysts Journal, vol. 54, no. 5, pp. 18-31.

16. SPIVA U.S. Scorecard Year-End 2014: S\&P Dow Jones Indices data [Electronic resource]. DOI: http://www.spindices.com/documents/spiva/spiva-us-year-end-2014.pdf.

17. Wermers, R. (2000), "Mutual fund performance: an empirical decomposition into stockpicking talent, style, transactions costs, and expenses", The Journal of Finance, vol. 55, no. 4, pp. 1655-1695. 